\title{
LA CONTABILIDAD NOMINAL EN ESPAÑOL Y BRIBRI DESDE LA PERSPECTIVA FUNCIONAL TIPOLÓGICA
}

\author{
Nominal countability in Spanish and Bribri from a functional typological perspective
}

Haakon Stensrud Krohn*

\begin{abstract}
RESUMEN
El presente artículo aborda las nociones de sustantivos contables e incontables en español y bribri desde un punto de vista funcional tipológico, según el cual se presume que la codificación morfosintáctica de la contabilidad constituye un reflejo icónico de la conceptualización del referente por parte del hablante. Las teorizaciones predominantes sobre el tema afirman que en las lenguas de clasificadores numerales, como el bribri, todos los sustantivos son permanentemente incontables, a diferencia de sus contrapartes en las variedades lingüísticas que no poseen clasificadores numerales; sin embargo, nuestra aproximación indica que las diferencias entre los dos tipos de lenguas en cuanto a la contabilidad nominal son mínimas.

Palabras clave: Español, bribri, contabilidad nominal, morfosintaxis, clasificadores numerales.
\end{abstract}

\begin{abstract}
This article focuses on the notions of countable and mass nouns in Spanish and Bribri from a functional typological perspective, which considers the morphosyntactic codification as an iconic reflection of the speaker's conceptualization of the referent. The dominant theories in the field assert that in numeral classifier languages, like Bribri, all nouns are always mass, unlike nouns in languages that don't have such classifiers. However, our approximation indicates that the differences between the two language types regarding the nominal countability are minimal.
\end{abstract}

Key Words: Spanish, Bribri, nominal countability, morphosyntax, numeral classifiers.

\footnotetext{
* Universidad de Costa Rica. Profesor Escuela de Filología, Lingüística y Literatura. Costa Rica.

Correo electrónico: hkrohn@gmail.com

Recepción: 12/07/15. Aceptación: 2/12/15.
} 


\section{Introducción}

El presente artículo constituye un análisis comparativo de la codificación morfosintáctica de la contabilidad nominal en español y bribri ${ }^{1}$, dos idiomas hablados en Costa Rica y Panamá. A pesar de la convivencia actual en estos países, se trata, desde luego, de lenguas tipológicamente muy distintas, originadas en dos continentes mutuamente alejados y pertenecientes a dos familias genéticas diferentes: la indoeuropea y la chibchense, respectivamente. El análisis se lleva a cabo en el marco de la Gramática Funcional Tipológica (GFT) de Givón (1983a, 1985, 2001a, 2001b, 2009), una teoría descriptiva y explicativa de las estructuras del lenguaje y de las lenguas.

El término contabilidad nominal alude a una dicotomía conformada por sustantivos (o sintagmas nominales) contables e incontables, un fenómeno ligado con la posibilidad de un referente nominal de ser cuantificado directamente como entidades discretas. La marcación morfosintáctica de la contabilidad se da como un reflejo de la manera que el hablante conceptualice y presente gramaticalmente al referente del sintagma nominal; como entidades individualizadas (contable) o como un concepto continuo (incontable). Las realizaciones gramaticales varían, por supuesto, de una lengua a otra, pero todas involucran cuantificadores y otras marcaciones del número nominal.

Evidentemente, los rasgos tipológicos más relevantes para este análisis se vinculan con las estrategias empleadas en cada lengua para cuantificar a un referente nominal. En lo que concierne a las dos variedades lingüísticas estudiadas, ambas presentan una marcación morfológica del número gramatical correspondiente a la distinción entre singular y plural. Sin embargo, la indicación explícita de plural es más frecuente en español, dado que se encuentra sometida a mayores restricciones semánticas y sintácticas en bribri. Además, ambas lenguas se diferencian en un punto importante relativo al uso de numerales: mientras que el español permite la cuantificación directa de un sustantivo por un numeral, el bribri requiere el empleo de un clasificador numeral, por lo que los numerales en la última lengua nunca se asocian directamente con un nombre. Como veremos en este artículo, la hipótesis más influyente en la actualidad afirma que esta diferencia se debe al hecho de que en las lenguas que presentan clasificadores numerales, todos los sustantivos siempre son incontables. Una de las finalidades del presente trabajo es proporcionar una perspectiva distinta, la cual implica mayor similitud en cuanto a la contabilidad nominal entre los dos tipos de lenguas.

En lo que respecta a la Gramática Funcional Tipológica, esta consiste en una redefinición "de los conceptos saussureanos de diacronía y sincronía por medio de la comparación y la generalización interlingüística" (Martín 1997: 281), dado que parte de la suposición de que existe un vínculo entre la forma lingüística y la función, tanto a nivel diacrónico como sincrónico. La gramática y la representación cognitiva constituyen los dos subsistemas básicos del lenguaje. A este respecto, un concepto fundamental es la iconicidad: la GFT presupone que la función expresada se refleja icónicamente en la gramática, lo cual quiere decir la gramática no es arbitraria (Givón 2001a: 34). Aun así, la iconicidad no es absoluta, sino gradual; una función puede ser expresada mediante más de una forma, y viceversa. Justamente estas elecciones por parte de los hablantes constituyen la base del cambio lingüístico. Además, la gramática cumple dos papeles en la GFT; aparte de codificar la semántica, también establece la coherencia discursiva (Givón 2001a: 399). En cuanto a la dimensión tipológica de la teoría, Becerra (2011: 113) señala que "un enfoque tipológico no supone desconocer las particularidades de las lenguas, sino valorarlas como expresión diversa de dominios funcionales humanos, enfrentados a contextos de uso reales."

En resumen, con el presente artículo pretendemos exponer, de manera clara y concisa, la forma en que las estructuras lingüísticas en 
español y bribri proyectan gramaticalmente la conceptualización de la naturaleza del referente por parte del hablante. Además, el análisis que proponemos para la contabilidad nominal en bribri es innovador en el sentido de que difiere de las teorizaciones predominantes sobre los idiomas de clasificadores numerales, las cuales se enfocan en la contabilidad como una propiedad sintáctica inherente e invariable de los sustantivos.

\section{Fundamentos semánticos}

Como hemos adelantado, en este artículo nos basamos en la premisa de que la distinción entre sustantivos contables e incontables a nivel morfosintáctico icónicamente refleja una diferencia de significado, una perspectiva apoyada por autores como Wierzbicka (1988), Borer (2005), Bale y Barner (2007), y Pelletier (2012). Por lo tanto, con el fin de dar cuenta de los factores que favorecen las distintas marcaciones gramaticales de la contabilidad, debemos empezar por examinar la semántica del fenómeno. Seguidamente, pasaremos a los análisis de la codificación gramatical en las dos lenguas en cuestión.

La dicotomía semántica entre sustantivos contables e incontables se basa, en primera instancia, en la naturaleza del referente. Esto es acertadamente explicado en la definición proporcionada por la Real Academia Española (2010: 211):

Los nombres contables (también llamados discontinuos y discretos) aluden a entidades que se pueden contar o enumerar (un libro, tres planetas, cuatro formas de proceder), mientras que los no contables (también denominados incontables, continuos, de materia, de masa y medibles) designan magnitudes que se interpretan

como sustancias o materias (un poco de café, demasiada testarudez, mucho tiempo). Aunque lo contable no es en realidad el nombre, sino las entidades que denota, el término nombre contable constituye una convención aceptada. Está, además, bien fundamentada, puesto que los nombres son contables no solo por la naturaleza de lo que designan, sino en función de su comportamiento gramatical. Así, el sustantivo información es contable en español (dos informaciones), pero no en inglés (*two informations).

Similarmente, de acuerdo con la gramática cognitiva de Langacker (1987, 1991), un sustantivo incontable es aquel que tenga un referente que se conceptualiza como una realidad continua e ilimitada, típicamente un líquido, una materia o un polvo. El término conceptualización es fundamental en este respecto, ya que la gramática no necesariamente codifica la realidad, sino una interpretación de la realidad en términos lingüísticos por parte del hablante.

Pelletier(2012:455) señala que la diferencia básica entre los dos tipos de referentes radica en dos propiedades estrechamente vinculadas. $\mathrm{La}$ primera es que los incontables, a diferencia de los contables, poseen la característica de referencia cumulativa, una noción establecida por Quine (1960). Formalmente, quiere decir que si dos o más referentes del mismo tipo se combinan, la suma se conceptualiza como exactamente lo mismo de lo que eran sus componentes. Esto se ilustra en el ejemplo (1), donde observamos que agua denota un referente incontable, ya que dos porciones de agua (de dos recipientes diferentes) que se echan en un mismo vaso se interpretan como una sola porción de agua. Lo mismo ocurre con ropa, por lo que ese referente también es codificado mediante un sustantivo incontable. En cambio, un perro y una idea son contables porque los dos referentes, cognitivamente, se mantienen separados aun cuando se tratan como un conjunto ${ }^{2}$.

$$
\begin{aligned}
& \text { a. agua }+ \text { agua }=\text { agua } \\
& \text { b. ropa }+ \text { ropa }=\text { ropa } \\
& \text { c. un perro }+ \text { un perro } \neq \text { un perro } \\
& \text { d. una idea }+ \text { una idea } \neq \text { una idea }
\end{aligned}
$$

No obstante, para autores como Gillon (1992, 1999), tal criterio no es apropiado para la identificación de sustantivos incontables, puesto que también la forma plural de los nombres contables presenta referencia 
cumulativa: el conjunto de varios grupos de perros igualmente se denomina perros ${ }^{3}$.

La segunda característica principal de los referentes incontables es la de referencia divisiva. En estos términos, una entidad incontable puede segmentarse en un número arbitrario de porciones, y cada porción se va a considerar como lo mismo que la totalidad. Por ejemplo, si el contenido de una jarra es cerveza, y esta se sirve en varios vasos, el contenido de cada vaso también es cerveza. En cambio, las partes de un referente contable como un perro, por ejemplo la lengua o las patas, no se consideran un perro.

Según señala Chierchia (1998), se suele hablar de la "atomicidad" del referente para aludir a su divisibilidad: un sustantivo contable denota un concepto atómico, o elemento mínimo de la extensión de la palabra, ya que la denotación de la palabra no incluye las partes constituyentes del referente, por ejemplo los componentes de un perro o el hecho de que una rama o una hoja de un árbol no es un árbol. En cambio, el referente de un sustantivo incontable no es el elemento mínimo de la extensión de la palabra, puesto que una parte de algo que es agua también puede considerarse agua, y una parte del referente de la palabra arena también puede denominarse de la misma forma.

Sin embargo, la referencia divisiva como criterio para discernir los referentes incontables de los contables también ha sido criticada, entre otros por Quine (1960: 98), quien detalla que existen "parts of water, sugar, and furniture too small to count as water, sugar, furniture" (tres sustantivos típicamente incontables en inglés). También Chierchia (1998: 68) recalca que debemos admitir que los sustantivos incontables, técnicamente, denotan conceptos atómicos cuando bajamos a un nivel muy detallado: un átomo de hidrógeno que forma parte de una molécula de agua no es agua, y un grano de arena no suele considerarse arena. Asimismo, el referente de un sustantivo incontable como ropa definitivamente posee partes mínimas que no son ropa. No obstante, como hablantes ignoramos este tipo de detalles ontológicos, ya que son irrelevantes para lo que queremos comunicar. Por ello, reiteramos, la base semántica de la distinción contable/incontable no reside en la forma ontológica exacta del referente, sino en la representación de esta en un mundo proyectado.

Finalmente, mencionaremos la perspectiva de Jackendoff (1991), que también será relevante para nuestro análisis. Este lingüista sostiene que la estructura interna no es el rasgo relevante para discernir entre los dos tipos de referentes. En cambio, afirma que la diferencia reside en el rasgo semántico que llama "boundedness", que aquí denominaremos delimitabilidad en español. Con este punto de vista, un referente contable es delimitable (y viceversa), ya que se conceptualiza como físicamente delimitado, a diferencia de los incontables, cuya dimensionalidad no es relevante.

\section{Codificación de la contabilidad nominal en español}

En esta sección nos centraremos en la manifestación morfosintáctica de la contabilidad nominal en español. Haremos uso del rasgo binario [contable], el cual es asignado el valor positivo cuando un sintagma nominal contiene alguna marcación morfosintáctica de que su referente es contable; de lo contrario, exhibe el valor negativo.

\subsection{Marcadores morfosintácticos de [+contable]}

El español posee varias estrategias morfosintácticas diferentes para expresar que un sintagma nominal alude a una entidad conceptualizada como contable. Una de esas es la cuantificación directa por medio de un numeral, como es el caso con manos y galletas en el ejemplo (2).
a. Tengo dos manos.
b. Me comí cuatro galletas.

También la marcación morfológica de plural constituye una marca del valor [+contable], puesto que solamente un referente 
discreto puede expresarse como plural en español. Adicionalmente, muchos de los cuantificadores indefinidos cumplen la misma función. Esto ocurre, entre otros, con muchos, pocos, algunos y varios (en su forma plural), además de ambos y cada; todos implican que el referente es constituido por varias entidades individualizadas $\mathrm{y}$, por lo tanto, es contable. De este modo, dichos cuantificadores, así como todos los números cardinales, funcionan como marcadores sintácticos del valor [+contable]. Allan (1980) se refiere a este conjunto de palabras como denumeradores (ing. "denumerators").

En cambio, al igual que muchas otras lenguas (Allan 1980: 545), el español no presenta marcadores morfosintácticos de la "incontabilidad", es decir, del valor [contable]. Como apreciaremos más adelante, la ausencia de marcación explícita de [+contable] no necesariamente significa que el referente sea [-contable] a nivel semántico, ya que el contexto físico o discursivo también influye en la interpretación de este rasgo. No obstante, la presencia de marcación del valor [+contable] siempre implica que el referente es conceptualizado de tal manera.

Como otro ejemplo concreto de la especificación morfosintáctica de la contabilidad, consideraremos el sustantivo carros en (3a), que es codificado como [+contable] por medio de la $-s$ de plural y cualquiera de los cuantificadores entre los corchetes. Dada la presencia del plural morfológico, ni siquiera hace falta un denumerador para interpretar el referente como [+ contable]. En contraste, agua en (3b) es [contable], debido a la ausencia de marcación plural y denumeradores (mucha y poca, que están en singular, no forman parte del conjunto de denumeradores en español, ya que se emplean con referentes continuos).

a. Juana tiene $\{$ dos/algunos $/$ muchos $/ \varnothing\}$
carros.
b. Pedro tomó $\{$ mucha/poca $/ \varnothing\}$ agua ayer.

No solo las construcciones en plural poseen el valor [+contable]; también el numeral uno y los artículos indefinidos cumplen la función de denumeradores, puesto que algo que se puede expresar como una unidad, necesariamente es contable. Esto se aprecia con árbol en el ejemplo (4a). Agua en (4b), por su lado, no acepta el artículo indefinido en la construcción proporcionada, ya que, en este caso, explícitamente alude a un líquido, cognitivamente representado como incontable.

(4) a.Un árbol es una planta.

b.*Un agua es un líquido potable.

En lo que concierne al determinante $c a d a$, el sustantivo al que se añade siempre aparece en singular, como se ilustra con día en (5). De todos modos, cada implica una cantidad de entidades discretas superior a uno, por lo que día es [+contable] en este caso.

Cada día salgo a correr.

\subsection{La variación}

Tradicionalmente, las gramáticas del español tratan los sustantivos como si fueran inherentemente contables o incontables, es decir, con un valor específico del rasgo [contable] codificado en el lexicón. Sin embargo, como vamos a apreciar, es muy inconveniente postular que la división se dé a ese nivel. Para ilustrar este punto, consideremos los sustantivos agua y arena. Pese a su función prototípica de incontables, existen varias estrategias por medio de las cuales se les puede especificar la cantidad. Una de ellas es el uso de algún término mensurativo, por ejemplo botella o kilo, como observamos en (6).
a. una botella de agua
b. dos kilos de arena

Aquí, agua y arena no son cuantificados directamente por el numeral. En cambio, los numerales hacen referencia a los términos mensurativos (que constituyen el núcleo de estos sintagmas nominales), por lo que estos aparecen codificados como contables y funcionan como delimitadores de agua y arena, que a su vez son 
incontables por aparecer en singular y no ser directamente enumerados.

El valor [-contable] de estos últimos sustantivos, el cual representa la idea de materias continuas con referencia cumulativa y divisiva, es evidente ya que no reciben marcación de plural. En contraste, manzanas y flores en (7) sí están en plural, de ahí que sí sean contables a pesar de que la palabra cuantificada por el numeral es el término mensurativo.
a. dos bolsas de manzanas
b. cuatro puños de flores

No obstante, los sustantivos prototípicamente incontables también pueden cuantificarse sin la presencia de un término mensurativo; una variedad de este fenómeno es conocido como el Empacador Universal (ing. Universal Packager), término establecido por Bach (1986). Este consiste en que el uso de un denumerador provoca que un referente normalmente continuo se conceptualice como empacado en contenedores o dividido en porciones discretas, como se ejemplifica en (8).

a. Me estoy tomando un café.

b. Dame dos aguas.

En estos casos, los sustantivos café y agua sí son enumerados directamente y, además, aparecen en plural si la cantidad es superior a uno. De ahí que se presenten como contables, lo cual refleja el hecho de que, en vez de aludir únicamente al contenido de los contenedores, hacen referencia al contenedor y el contenido en conjunto, típicamente tazas o botellas, las cuales constituyen entidades discretas.

Un fenómeno muy similar es conocido como el Organizador Universal (ing. Universal Sorter) (Bunt 1985). La diferencia respecto al Empacador Universal es que, en estas ocasiones, las individualizaciones constituyen agrupaciones abstractas, como en el ejemplo (9), donde vinos implica tipos o marcas de esta bebida, sin referirse a porciones concretas.

(9) Los mejores vinos son de España.

También se puede dar el efecto contrario, mediante un fenómeno denominado Picadora
Universal (ing. Universal Grinder) (Pelletier 1975). Esto sucede cuando un sustantivo prototípicamente contable se emplea como incontable, tal como se aprecia en el ejemplo (10). En (10a), tomates es cuantificado y lleva un sufijo de plural, por lo que es contable. En cambio, en (10b), dicho sustantivo se presenta como [-contable], una representación icónica de la irrelevancia de presentar este referente como entidades discretas cuando está picada y funciona como ingrediente.

(10) a. Piqué dos tomates.

b. Hay tomate en la ensalada.

Asimismo, queque es especificado (por medio del numeral y el sufijo de plural) como contable en (11a), pero se usa como sustantivo incontable en (11b), ya que el número de entidades discretas no se considera relevante para lo que se desea comunicar.
a. Ayer comimos dos queques.
b. Ayer comimos queque.

Lo mismo sucede con carro (nuevo) en el siguiente ejemplo, donde es contable en (12a) e incontable en (12b). En el primer caso alude a un referente concreto y específico, mientras que en el segundo hace referencia al concepto general de "carro nuevo".

(12) a. Ayer compré un carro nuevo.

b. Aquí huele a carro nuevo.

También los sustantivos con referentes abstractos pueden emplearse con ambos valores, como podemos apreciar con diferencia en (13), en el que es incontable en (13a) y contable en (13b).

(13) a. No había mucha diferencia entre las dos propuestas.

b. Había dos diferencias principales entre las dos propuestas.

Incluso un sustantivo prototípicamente incontable como calor puede ser codificado como contable; esto se observa en el ejemplo (14), donde tal marcación se debe a la interpretación 
de este como distinto de otros tipos de calor, de ahí que se presente como una entidad discreta.

a. Ayer hizo un calor insoportable.

b. Los calores de la menopausia son más frecuentes en la noche.

Asimismo, la forma plural de aguas en enunciados como el que se presenta en (15) también corresponde a una separación conceptual de este referente respecto a otras aguas.

(15) Navegamos por las aguas del Nilo.

De los ejemplos anteriores podemos concluir que una gran cantidad de los sustantivos españoles pueden ser tanto contables como incontables, dependiendo de cómo el hablante conceptualice al referente. De hecho, probablemente todos podrían utilizarse en ambos sentidos, dado el contexto adecuado. A este respecto, Pelletier (2012) señala que cualquier objeto podría pasarse por una picadora enorme (la Picadora Universal): si lo hiciéramos con un sombrero, el piso podría llenarse de sombrero; con un elefante, habría elefante por todas partes. En consecuencia, clasificar a los nombres como contables o incontables a nivel léxico resultaría, prácticamente, en una duplicación del número de entradas léxicas. Por eso, debemos postular que el rasgo [contable] se especifica a la hora de usar el sustantivo en un enunciado. En alusión a esto, Rothstein (2010: 19) señala:

The mass/count distinction can only be explained in terms of how the expressions refer, and not in terms of the things they refer to. This means it is a grammatical and not an ontological distinction.

Dado que el valor del rasgo [contable] es determinado por la presencia de una flexión plural o un denumerador, cuyo alcance comprende todo el sintagma nominal, debemos reconocer, al igual que autores como Bunt (1976) y Allan (1980), que dicho valor se les especifica a los sustantivos a nivel sintagmático. De este modo, el núcleo de un sintagma nominal es contable si el sintagma en sí es contable, y viceversa. Por lo mismo, hablamos en este artículo de la "contabilidad nominal" en vez de la "contabilidad de sustantivos", aunque el último es el término más recurrente en la literatura sobre el tema. Bunt (1976: 81) defiende nuestro punto de vista de la siguiente manera:

$[\mathrm{T}]$ he count/mass distinction is not really a distinction between words, but a distinction between ways of using words. [...] The count/mass distinction can be drawn not only between bare nouns, but also between complex noun phrases. In what follows, when we speak of mass nouns, this has to be understood as short for: noun phrases, used in a mass sense.

A este respecto, es importante recordar que un sintagma nominal puede estar subordinado a otro, con el valor contrario del rasgo [contable]. Por ejemplo, el sintagma nominal una botella de agua que vimos en (6a) es contable, lo cual es indicado por la palabra una, que alude al núcleo, botella. En cambio, aunque agua forma parte de este sintagma contable, también constituye un sintagma nominal por sí solo (que se encuentra dentro de un sintagma preposicional), que a su vez es incontable.

Existen varias teorías recientes que abogan por evitar la especificación del rasgo [contable] a nivel léxico, de modo que este se especifique a la hora de formar un sintagma nominal, tal como argumentamos en el este artículo; entre ellos se encuentran Borer (2005), Bale y Barner (2007) y Pelletier (2012). Las diferencias entre estos autores radican en el rol que le conceden a la semántica. Mientras la teoría de Borer (2005) es puramente sintáctica, sin ningún aspecto semántico, los otros dos distinguen entre la contabilidad semántica y sintáctica, igual que hacemos en el presente trabajo. De estos, Pelletier considera que todos los sustantivos llevan ambos rasgos [+contable] y [ + masa] (lo equivalente a [-contable]) en el lexicón, y que uno de estos se elide al usar la palabra en una oración. Bale y Barner (2007), por su lado, afirman que los sustantivos no presentan ningún valor del rasgo [contable] antes de que este sea especificado por la morfosintaxis.

\subsection{El contexto como marcador de la contabilidad}

Ya hemos señalado que los artículos indefinidos del singular funcionan como 
marcadores del valor [+contable] en español. Sin embargo, con los artículos definidos del singular no ocurre lo mismo. Consideremos los siguientes ejemplos:

\section{(16) a. Gerardo estuvo en la cárcel.}

b. Gerardo estuvo en prisión.

En (16a), la cárcel puede representar dos referentes distintos: si el emisor quiere comunicar que el sujeto estuvo un tiempo (tal vez solo unas horas) en una cárcel específica, que constituye un referente discreto, el sintagma denota una entidad semánticamente contable. A su vez, si el referente es alguien que pasó años tras las rejas en varias cárceles diferentes, el sustantivo alude al concepto general de cárcel, el cual es semánticamente incontable. En cambio, con prisión en (17b) no se da la misma ambigüedad, pues este sustantivo aparece sin el artículo (que es el elemento que permite las dos lecturas) y, por lo tanto, solamente se puede entender en el sentido incontable.

Los ejemplos en (17) son similares. $E l$ león en (17a) denota claramente una entidad discreta si el enunciado es emitido, por ejemplo, por una persona que está señalando un león ubicado entre animales de otras especies más pequeños y amigables. No obstante, la misma oración se puede pronunciar sin que el león haga alusión a ningún individuo en específico, sino a la especie en general, caso en el que es semánticamente incontable. Por su lado, el polvo en (17b) es necesariamente incontable, ya que es evidente que no refiere a una entidad discreta. Aun así, admite dos lecturas: puede entenderse bien como el polvo en general, o bien como una cantidad de polvo determinada que está presente mientras el hablante emite el enunciado.

\section{a. El león es grande y peligroso. \\ b. El polvo me produce alergia.}

A partir de (16) y (17) podemos concluir que el artículo definido del singular no funciona como marcador inequívoco del rasgo [+contable], sino que, más bien, deja el rasgo [contable] sin especificar. De todos modos, el referente no necesariamente es conceptualizado como incontable en el último caso. La falta de marcación se debe al hecho de que, cuando está presente este artículo, la sintaxis española no permite la presencia de ningún denumerador en el sintagma nominal. En consecuencia, aunque el emisor del enunciado (17a) tenga un león específico y discreto en mente, el rasgo semántico [+contable] no siempre aparece codificado morfosintácticamente.

La razón por la que los artículos definidos del singular ocasionan esta ambigüedad es, evidentemente, porque en la vida real, un enunciado como (17a) nunca se pronunciaría fuera de todo contexto. Los artículos definidos, a diferencia de los indefinidos, tienen una función deíctica o anafórica; es decir, implican que el referente es conocido por parte del destinatario. Por ello, no hace falta especificar gramaticalmente la contabilidad del referente. La ausencia de la especificación del rasgo [contable] en los sintagmas nominales con artículo definido del singular constituye, por tanto, una economización en la lengua; puesto que el referente es conocido, nunca va a ser ambiguo.

Consideremos ahora el ejemplo (18), donde el referente de la pintura claramente es incontable y específico (gracias a la precisión brindada por el sintagma preposicional).

(18) La pintura en la pared ya se secó.

Si nos volvemos a fijar en la lectura genérica de el león en (17a), es decir, cuando este nombre alude a los leones en general, observamos que no se trata del mismo tipo de referencia que la de la pintura en (18). Mientras que la pintura denota un referente en el mundo real, a saber, toda la pintura que está en una determinada pared, el león (en su sentido genérico) no hace referencia a un león en específico, ni a todos los leones que existen, ya que por ejemplo hay leones bebés que no son ni grandes ni peligrosos; más bien, alude al león prototípico, es decir, un concepto abstracto que posee los rasgos más típicos de este animal. Lo mismo sucede con la lectura genérica de el polvo en (17b) ("el polvo en general"). Tal hecho pone en evidencia que las referencias genéricas son incontables, pero que un sintagma 
nominal incontable no necesariamente presenta referencia genérica.

En cuanto a los demostrativos en singular, estos tampoco especifican ningún valor del rasgo [contable], dado que pueden usarse con un referente contable, como en (19a), o incontable, como en (19b). No obstante, a diferencia de los artículos definidos, los demostrativos nunca permiten una lectura genérica.
a. Este carro está sucio.
b. Esta agua está sucia.

\subsection{Sustantivos que presentan menor grado de variación}

Ahora bien, nuestra perspectiva funcionalista no socava el hecho de que algunos sustantivos, por ejemplo agua, grasa y polvo, debido a la naturaleza de sus referentes, se utilizan como incontables con mucha mayor frecuencia que nombres como casa, gato y pensamiento. En este sentido, estamos hablando de un continuum de contabilidad en el que los nombres que prácticamente siempre se utilizan como contables se encuentran en un extremo; y los que casi nunca funcionan de tal manera, en el otro. Con tal aproximación, un sustantivo como casa se ubicaría cerca del extremo contable del continuum, mientras que un sustantivo típicamente incontable que en ciertos contextos se utiliza como contable, tal como agua o vino, se situaría más cerca del centro de la escala. Cerca del extremo incontable estarían los sustantivos singularia tantum, es decir, los que casi siempre aparecen en singular, por ejemplo sed, importancia y constitucionalidad. Estos representan conceptos de tal naturaleza que difícilmente pueden interpretarse como discretos, salvo en casos como los que apreciamos en el ejemplo (14). Lógicamente, la ubicación de cada referente en el continuum está motivada por sus características ontológicas, pero estas siempre pueden ser interpretadas y proyectadas de manera diferente por parte del hablante. Por consiguiente, el Empacador Universal, el Organizador Universal y la Picadora Universal no representarían ningún cambio semántico en el sustantivo, sino que solamente constituyen usos menos prototípicos.

En español también hay un grupo de sustantivos que prácticamente siempre presentan el valor [+contable]. Se trata de los denominados pluralia tantum, que se caracterizan por no utilizarse en singular; algunos ejemplos son entrañas, nupcias, comestibles, viveres, plácemes, redaños, afueras y alrededores. Varios de ellos pueden asociarse con un denumerador, como se observa en (20). No obstante, este no es el caso con por ejemplo afueras y alrededores.

(20) a. El mes pasado hubo dos nupcias.

b. Llevaremos muchos comestibles.

La obligatoriedad de la marcación plural en este tipo de sustantivos parece deberse al hecho de que sus referentes consisten en varias entidades o eventos diferentes que, de acuerdo con Wierzbicka (1988: 540), han recibido un nombre común "because they are all in the same place, at the same time, and [...] they are in that place at that time for the same reason". Asimismo, el rechazo a los denumeradores por parte de algunos sustantivos pluralia tantum sugiere que, en estos casos específicos, la marcación de plural y los denumeradores aluden a dos niveles distintos: el plural, a los componentes; y los denumeradores, al referente en su totalidad.

\section{Codificación de la contabilidad nominal en bribri}

Después del análisis de la contabilidad nominal en español, realizaremos ahora un estudio similar del mismo concepto en bribri, idioma que presenta dos diferencias tipológicas importantes en comparación con la lengua indoeuropea: en primer lugar, la aparición de marcación morfológica de plural en bribri es mucho más restringida $\mathrm{y}$, en segundo lugar, posee una serie de clasificadores numerales que aparecen obligatoriamente junto con los numerales. 


\subsection{Características gramaticales relevantes}

Primeramente, presentaremos los rasgos gramaticales relevantes para este análisis, a saber, las diferentes estrategias que posee el bribri para expresar el número nominal.

\subsubsection{Marcación morfológica de plural}

El bribri distingue gramaticalmente entre singular y plural. El plural es el número morfológicamente marcado, y la lengua dispone de varias estrategias diferentes para indicar que un referente nominal es plural. Por razones de espacio, aquí veremos solo las más frecuentes, ya que el análisis en sí no requiere de un conocimiento exhausto. En Krohn (por publicarse), todas son detalladamente explicadas.

En primer lugar, el bribri presenta un sufijo $-p a^{4}$ que se agrega a un sustantivo cuyo referente es plural. Sin embargo, el uso de dicho morfema está condicionado por una jerarquía universal de empatía o animicidad: únicamente los sustantivos con referente humano lo reciben. Por lo tanto, los nombres en (21a-b) aceptan este sufijo, mientras que los de (21c-d) no lo suelen admitir. Por la misma razón, los dos últimos resultan ambiguos en cuanto al número cuando aparecen descontextualizados.
a. alákölpa 'mujeres'
b. yếriapa 'cazadores'
c. chìchi 'perro(s)'
d. ù 'casa(s)'

Además, cuatro adjetivos bribris presentan una forma plural que consiste en una reduplicación de la raíz (o de una raíz diferente). Esta marcación de número concuerda con la cantidad de la entidad calificada y puede utilizarse con cualquier referente, sea humano, animal o inanimado. Los cuatro adjetivos con sus respectivas formas se dan en la tabla 1.
TABLA 1.

Adjetivos bribris que presentan una forma plural

\begin{tabular}{llll}
\hline Singular & & Plural & \\
\hline bua' & 'bueno' & bua'bua' & 'buenos' \\
\hline buáala & 'bonito' & buàmbuáala & 'bonitos' \\
\hline bè̀rie & 'grande' & bulùbulù & 'grandes' \\
\hline 'pequeño' & tsìr & tsìrtsir & 'pequeños' \\
\hline
\end{tabular}

De esta manera, aunque el sustantivo ù 'casa' no acepta el sufijo -pa, el adjetivo proporciona la distinción entre singular y plural del referente en (22).

(22) a. Ù dör bềrie.

casa COP grande

'La casa es grande.'

b.Ù dör bulùbulù.

casa COP grande.PL

'Las casas son grandes.'

Asimismo, el bribri posee una serie de palabras denominadas posicionales. Estas aparecen en el predicado de construcciones locativas básicas o existenciales/presentativas. Al igual que los adjetivos, presentan una forma singular y una plural, y hacen referencia tanto a la posición o la postura como al número gramatical del referente nominal. La tabla 2 incluye todos los posicionales (menos uno, que no distingue el número) con sus traducciones aproximadas propuestas por Jara y García (2013: 103).

TABLA 2.

Posicionales en bribri

\begin{tabular}{lll}
\hline Singular & Plural & $\begin{array}{l}\text { Significado } \\
\text { aproximado }\end{array}$ \\
\hline dur & iề tẽn & de pie, parado, erguido \\
\hline tchër & tulur & $\begin{array}{l}\text { sentado, posado, } \\
\text { puesto }\end{array}$ \\
\hline tẽr & tulur & $\begin{array}{l}\text { metido, enterrado, } \\
\text { clavado }\end{array}$ \\
\hline
\end{tabular}




\begin{tabular}{lll}
\hline tër & tchề tẽn & tirado, tendido, echado \\
\hline ar & tchềnĩk & $\begin{array}{l}\text { colgado, suspendido, } \\
\text { flotando }\end{array}$ \\
\hline bàtsul & bàtsulur & $\begin{array}{l}\text { apoyado, pegado } \\
\text { verticalmente }\end{array}$ \\
\hline mẽr & dapárke & $\begin{array}{l}\text { puesto/acostado en } \\
\text { algo elevado }\end{array}$ \\
\hline
\end{tabular}

La distinción del número nominal por medio de un posicional se ejemplifica en (23), donde este elemento constituye la única marcación del número del referente de pùs 'gato'.
a. Pùs tër î́s kĩ.
gato echado suelo en
'El gato está echado en el suelo.'

b. Pùs tchềtẽn î́s kĩ.

gato echado.PL suelo en

'Los gatos están echados en el suelo.'

\subsubsection{Cuantificadores indefinidos}

La mayoría de los cuantificadores indefinidos bribris, al igual que los del español, implican que el referente nominal consiste en un conjunto de entidades discretas. Los más comunes de estos se presentan en la tabla 3.

TABLA 3.

Cuantificadores indefinidos bribris que implican plural

\begin{tabular}{ll}
\hline wồ kĩla & 'pocos' \\
\hline wếlè & 'algunos' \\
\hline tsée & 'muchos' \\
\hline tấ ĩ & 'muchos' \\
\hline ulìtãnẽ & 'todos' \\
\hline kốs & 'todos'
\end{tabular}

Los indefinidos se combinan directamente con el sustantivo, como observamos en (24). a. Ye' tö tsawì sãwế tsée.

1SG ERG armadillo ver-PRC muchos

'Yo vi muchos armadillos.'

b. Ye' tö tsawì ulìãnẽ sãwế.

1SG ERG armadillo todos ver-PRC

'Yo vi todos los armadillos.'

\subsubsection{Numerales}

Los cuantificadores numerales constituyen la única estrategia para expresar el número exacto de un referente en bribri. El idioma posee raíces numerales del uno al diez, los cuales se pueden combinar para crear números mayores. Además, el bribri es una lengua de clasificadores numerales; esto quiere decir que cada vez que se utiliza un numeral (con la excepción de 'diez' y, en la mayoría de los casos, 'nueve'), aparece un elemento adicional, denominado clasificador, el cual alude a una o varias propiedades de la entidad cuantificada. En bribri, el clasificador se agrega al numeral como sufijo. Es posible que el inventario de clasificadores varíe según la variedad dialectal, pero en el bribri de Coroma, Krohn (2014) identifica once morfemas clasificadores diferentes (de los cuales dos son homófonos) en el ámbito nominal. Estos pueden agruparse según su referencia: los que se aluden a una o varias propiedades individuales del referente se consideran sortales, y los que hacen referencia a las características de conjuntos del referente son denominados mensurativos.

Los numerales junto con los diferentes clasificadores se presentan en las siguientes tablas (basadas en Krohn 2014: 216); la tabla 4 incluye los sortales y la tabla 5, los mensurativos. Nótese que los morfemas que expresan las categorías de PLANTA EN PIE y UNIDAD DE PESO son homófonos, pero con significados claramente diferentes. Las palabras en itálica no llevan el sufijo correspondiente, lo cual se debe a uno de dos factores: o es una forma supletiva tomada de la serie humana, lo cual es el caso en todos los numerales en itálica menores de 'ocho', o no lleva ningún clasificador (o un sufijo cero), lo cual 
ocurre con las palabras en itálica con valores de 'nueve' o 'diez'. El hecho de que algunas casillas del cuadro están vacías se debe a que esas combinaciones de numeral+clasificador fueron consideradas agramaticales por el informante; lo mismo sucede con la raíz 'diez' con cualquiera de los clasificadores mensurativos.

TABLA 4

Numerales bribris del uno al diez con los diferentes clasificadores sortales

\begin{tabular}{llllll}
\hline & Humano & Redondo & Alargado & $\begin{array}{l}\text { Plano/ } \\
\text { abstracto }\end{array}$ & Planta en pie \\
\hline & -1 & $-\mathrm{k}$ & - töm & -t & -lka \\
\hline 1 & èköl & èk & ètöm & èt & élka \\
\hline 2 & bốl & bò̀ $\mathrm{k}$ & bồ töm & bồ t & bốlka \\
\hline 3 & mañál & mañál & mañàtöm & mañàt & mañálka \\
\hline 4 & tchếl & tchếl & tchềtöm & tchếl & tchếlka \\
\hline 5 & skếl & skếl & skềtöm & skếl & skếlka \\
\hline 6 & tèröl & tèröl & tèktöm & tèröl & \\
\hline 7 & kúl & kúl & kùktöm & kúl & \\
\hline 8 & pàköl & pàköl & pàktöm & pàköl & \\
\hline 9 & sũlì̀tu & sũlì̀tu & sũlì̀töm & sũlì̀tu & \\
\hline 10 & dabòm & dabòm & dabòm & dabòm & \\
\hline
\end{tabular}

Tabla 5.

Numerales bribris del uno al nueve con los diferentes clasificadores mensurativos

\begin{tabular}{|c|c|c|c|c|c|c|}
\hline & $\begin{array}{l}\text { Racimo de } \\
\text { banano }\end{array}$ & $\begin{array}{l}\text { Racimo } \\
\text { de } \\
\text { pejibaye }\end{array}$ & Puño & $\begin{array}{l}\text { Paquete/ } \\
\text { Bulto }\end{array}$ & Especie/clase & $\begin{array}{l}\text { Unidad de } \\
\text { peso }\end{array}$ \\
\hline & -yuwak & -tsõk & -yök & -kua & -ltë & $-1 \mathrm{ka}$ \\
\hline 1 & ètyuwak & étsõk & èyök & étkua & éltë & élka \\
\hline 2 & bồ tyuwak & bồ tsõk & bồyök & bồ tkua & bố ltë & bố lka \\
\hline 3 & mañátyuwak & mañàtsõk & mañàyök & mañàtkua & mañáltë & mañálka \\
\hline 4 & tchế lyuwak & & tchếyök & tchế tkua & tchếltë & tchế lka \\
\hline 5 & skếlyuwak & & skếyök & skềtkua & skếltë & skếlka \\
\hline 6 & & & tèryök & & & \\
\hline 7 & & & kúlyök & & & \\
\hline 8 & & & pàryök & & & \\
\hline 9 & & & sũlì̀tuyök & & & \\
\hline
\end{tabular}




\subsection{Función de los clasificadores numerales}

Abajo se dan ejemplos de los usos de los dos tipos de clasificadores; sortales en (25) y mensurativos en (26). Como se aprecia, ambos tipos de clasificadores pueden utilizarse sin importar la naturaleza del referente cuantificado: los ejemplos (25a-b) y (26a-b) incluyen sustantivos que en español son codificados como individualizados, mientras que los nombres en (25c-d) y (26c-d) denotan sustancias típicamente expresadas como incontables en español.
a. ũ̀ èk
olla uno-CLRED
'una olla'
b. di' tchềtöm
agua cuatro-CLALA
'cuatro ríos'
c. alákölpa bố 1
mujer dos-CLHUM
'dos mujeres'
d. tabè chikà mañàt
machete material tres-CLPLA
'tres entidades planas de metal'

a. mamawö èyök

flor uno-CLPUÑ

'un puño de flores'

b. chãmù bồ tyuwak

banano dos-CLRBA

'dos racimos de banano'

c. kiố mañàtkua

manteca tres-CLPAQ

'tres paquetes de manteca'

d. asùkar tchế lka

azúcar cuatro-CLPES

'cuatro unidades de peso (kilos) de azúcar'

Es importante señalar que, en las construcciones con numerales, el clasificador no solo es obligatorio sino que también funciona como núcleo semántico del sintagma nominal. Esto se nota, en primer lugar, por el hecho de que el elemento directamente cuantificado es el clasificador, no el sustantivo: por esa razón, la traducción de (26b) no es 'un racimo de dos bananos', sino 'dos racimos de banano'. En segundo lugar, si se incluye un adjetivo, este expresa una cualidad del concepto codificado por el clasificador, lo cual se evidencia en (27), donde bulùbulù 'grandes' hace referencia a los racimos, no a los bananos.

chãmù bồ tyuwak bulùbulù

banano dos-CLRBA grande-PL

'dos racimos grandes de bananos'

Cabe subrayar que ningún sustantivo es inherentemente asociado con un clasificador determinado; la variación en el uso de estos sufijos constituye una herramienta productiva para especificar o ajustar el significado de los nombres. Puesto que cada clasificador, en teoría, podría asociarse con cualquier sustantivo, no existe fundamento para postular que los sortales funcionan de manera diferente de los mensurativos. El papel de los clasificadores como núcleo semántico del sintagma nominal parece ser una característica compartida por todas las lenguas del mundo que presentan este tipo de elementos. Como señala Yi (2009: 210), tal hecho ha llevado a un gran número de autores a concluir que en estas lenguas, todos los sustantivos son incontables de manera constante; es decir, que presentan el valor [-contable] tanto a nivel del lexicón como a nivel sintagmático. Por lo tanto, los clasificadores, que serían los únicos elementos contables, se incluirían para permitir la cuantificación de los nombres, de manera muy similar a los términos mensurativos en español. Esta suposición es resumida en la llamada Hipótesis de Sustantivos de Masa (HSM) (ing. Mass Noun Hypothesis), según la cual (a) las lenguas de clasificadores no poseen sustantivos contables y (b) todos los sustantivos comunes en tales lenguas son (invariablemente) sustantivos incontables.

De este modo, todos los clasificadores funcionarían comoelementos "individualizadores" de conceptos generales expresados por los sustantivos, hecho que explicaría por qué los clasificadores se presentan obligatoriamente a la hora de utilizar un numeral. Dicho de otra manera, en las construcciones numerales en estas 
lenguas, los sustantivos siempre constituirían referencias genéricas; aludirían a las propiedades de un prototipo abstracto, concebido como incontable, y necesitarían de un clasificador para ser individualizados. Las glosas más precisas para los ejemplos (25a-b) serían, entonces, las que se proporcionan en (28).

$$
\begin{aligned}
& \text { a. û̀ èk } \\
& \text { olla uno-CLRED } \\
& \text { 'una entidad redonda de (el concepto de) } \\
& \text { olla' } \\
& \text { b. di' tchề töm } \\
& \text { agua cuatro-CLALA } \\
& \text { 'cuatro entidades alargadas de (el concepto } \\
& \text { de) agua' }
\end{aligned}
$$

Cuando el clasificador es una forma supletiva, la referencia sería menos específica, como se propone en (29), donde la forma tchếl se repite en la serie de cuatro clasificadores diferentes (cf. tabla 4).

\section{(29) àsh tchế1}

cítrico cuatro-CLHUM

'cuatro entidades humanas/redondas/ planas/abstractas de cítrico'

No obstante, hemos apreciado que los numerales 'nueve' y 'diez' se presentan sin sufijo. En cuanto a la interpretación cognitiva en estos casos, habría dos análisis posibles. Uno es que el morfema supletivo o cero se entiende como ' $\mathrm{X}$ entidades de', sin especificación de ninguna otra propiedad. La otra posibilidad es que estos numerales cuantifican directamente al sustantivo, al igual que los cuantificadores indefinidos, pero a diferencia de los demás numerales.

Ahora bien, mostramos en la sección 4.1. que el bribri posee varias estrategias para expresar el número plural de un sustantivo. Con la excepción de los numerales, ninguna de estas evoca la presencia de un clasificador, lo cual constituye un gran reto a la HSM, ya que difícilmente se puede postular que los sustantivos son permanentemente incontables si pueden ser flexionados para número sin este elemento "individualizador" adicional. Además, los sustantivos con referente humano exhiben flexión de plural también cuando se encuentran en una construcción numeral, como apreciamos en el ejemplo (25c). Esto contradice por completo la suposición de que los clasificadores son obligatorios debido a una necesidad de individualizar al referente del sustantivo; por lo contrario, la flexión de plural muestra que el sustantivo perfectamente puede representar una entidad discreta sin la ayuda de un clasificador. En el siguiente apartado examinaremos esta observación con mayor detalle.

\subsection{Marcadores morfosintácticos de [+contable]}

En los últimos años, varios autores han expuesto evidencias en contra de la HSM, afirmando que ciertas lenguas de clasificadores numerales sí hacen una distinción entre sustantivos contables e incontables. Por ejemplo, Yi (2009) sostiene que los nombres en chino mandarín que pueden asociarse con el clasificador ge, el cual conlleva un sentido individual, son contables, mientras que los demás son incontables. Similarmente, Zhang (2012) defiende la idea de que es la posibilidad de ser modificado por adjetivos relacionados con dimensionalidades físicas ('grande', 'pequeño', 'largo', 'corto', 'ancho', 'estrecho', etc.) lo que define a un sustantivo contable (a nivel léxico) en esa lengua. ${ }^{5}$

Dado que una distinción gramatical entre número singular y plural implica la existencia de sustantivos contables, la HSM se fundamenta en la suposición de que las lenguas de clasificadores numerales no poseen flexiones de plural. De acuerdo con Zhang (2012: 78), dicha presunción tiene sus raíces en Greenberg (1974), quien afirma que "numeral classifier languages generally do not have compulsory expression of nominal plurality, but at most facultative expression" (p. 25). Posteriormente, esta generalización se habría convertido en una distribución complementaria entre clasificadores numerales y marcadores de plural en las influyentes obras de T'sou (1976), 
Doetjes (1996, 1997) y Chierchia (1998), lo cual, como hemos comprobado, es falso en el caso del bribri. También Allan (1977: 294) y Aikhenvald (2003: 100-101) incluyen ejemplos de lenguas en las que convive la marcación de plural con clasificadores numerales. Asimismo, Zhang (2012: 80) muestra que en mandarín, el plural puede ser expresado por medio de una reduplicación del clasificador y que en indonesio (otra lengua de clasificadores numerales), la misma función se lleva a cabo reduplicando el sustantivo.

Volviendo al bribri, la flexión de plural asociada con los nombres en las oraciones en (30) constituye, claramente, un indicador del valor [+contable].

(30) a. Awápa tsë’.

médico-PL cantar-PRC

'Los médicos cantaron.'

b. Ye' tö ù bulùbulù sãwề.

1SG ERG casa grande-PL ver-IMP

'Veo las casas grandes.'

c. Chìchi iềtẽn.

perro de.pie.PL

'Los perros están de pie.

Incluso la forma singular del posicional en (31) señala que el referente es contable, ya que los posicionales no pueden aparecer con referentes interpretados como conceptos continuos. ${ }^{6}$

(31) Wếm tër î́s kĩ.

hombre echado.SG suelo en

'El hombre está echado en el suelo.'

Asimismo, los cuantificadores indefinidos difieren fundamentalmente de los numerales en el sentido de que los primeros sí se combinan directamente con el sustantivo, sin la aparición de un clasificador. Por ello, los sustantivos en el ejemplo (32) son marcados como contables por medio del cuantificador.
a. ák tsée
piedra muchos
'muchas piedras'

\section{b. talók ulìtãne \\ cocodrilo todos \\ 'todos los cocodrilos'}

Hemos mencionado brevemente que Zhang (2012) define los sustantivos contables como los que a nivel sintagmático pueden ser modificados por algún adjetivo que exprese una característica espacial propia de los referentes de límites precisos; estos los denominaremos "adjetivos delimitadores". Aunque la visión de [contable] como un rasgo codificado en el lexicón no es compatible con nuestra aproximación, sí acordamos en que los adjetivos delimitadores solo pueden aparecer con un sustantivo cuyo referente sea interpretado como discreto. Por lo tanto, tomaremos en cuenta el empleo de estas palabras como otro mecanismo para especificar un sustantivo como [+contable]. ${ }^{7}$ Los referentes nominales en (33) serían, entonces, inequívocamente contables.

$$
\begin{aligned}
& \text { (33) a. kàl kốjkẽ̀ } \\
& \text { árbol alto } \\
& \text { 'árbol alto' } \\
& \text { b. di' batsĩ̀ } \\
& \text { agua largo } \\
& \text { 'río largo' }
\end{aligned}
$$

Por último, es importante señalar que también un sustantivo en un sintagma que incluye un numeral puede ser marcado como [+contable], siempre y cuando su referente sea humano. Ya apreciamos el mismo fenómeno en (25c); (34) incluye dos más.
(34) a. bùsipa bố 1
muchacha-PL dos-CLHUM
'dos muchachas'
b. yëriapa mañál
cazador-PL tres-CLHUM
'tres cazadores'

Si tomamos en cuenta que el clasificador constituye el núcleo semántico, las glosas más literales de estos ejemplos serían 'dos humanos de muchachas' y 'tres humanos de cazadores', respectivamente. 


\subsection{Sustantivos no marcados como [+contable]}

El hecho de que los sustantivos en la mayoría de los casos no son marcados morfosintácticamente como contables cuando aparecen con un numeral, no necesariamente se debe, por lo tanto, a una conceptualización de sus referentes como conceptos continuos o abstractos, como afirma la HSM, sino a las restricciones que existen en el bribri para el uso de la marcación morfológica de plural. Por esta razón, un referente no humano puede ser concebido como contable por parte del hablante $\mathrm{y}$, aun así, aparecer sin marcadores que indiquen tal característica. Esto no sucede solamente con la presencia un numeral, sino en todas las ocasiones en las que la gramática no permite la expresión de tal propiedad explícitamente, a saber, cuando el referente no es humano y la cláusula no contiene ningún elemento pluralizable (es decir, un posicional o un adjetivo con forma plural, o bien mediante alguna de las otras estrategias que no hemos presentado en este artículo) ni un cuantificador indefinido. En el ejemplo (35) no se explicita si sàl 'mono colorado' $\mathrm{y}$ kàl wồ 'fruta (de árbol)' denotan referentes singulares o plurales, por lo que la oración puede significar 'El mono colorado comió una fruta.', 'Los monos colorados comieron una fruta.', 'El mono colorado comió frutas.' o 'Los monos colorados comieron frutas'.

Sàl tö kàl wồ katé.

mono.colorado ERG árbol fruta comer-PRC

Como se observa a continuación, los referentes discretos en (35) se presentan exactamente de la misma manera que los continuos en (36), sin recibir ninguna marcación adicional.

(36) Di’ tso' tchõ' kĩ. agua EST guacal en 'Hay agua en el guacal.'

b. Be' tö tấĩ ãlồ yé.

2SG ERG mucha chicha beber-PRC

'Tomaste mucha chicha.'
Las restricciones a las que se encuentra sometida la marcación de plural, sobre todo la del uso de -pa únicamente con referentes humanos, parece reflejar el hecho de que en bribri es menos relevante expresar gramaticalmente el número nominal que en español, especialmente si el referente no es humano. No se trata de un fenómeno inusual a nivel interlingüístico, ya que, como apunta Corbett (2000), la jerarquía de empatía/animicidad se manifiesta de manera similar en lenguas de todas las partes el mundo. La frecuente ausencia de la marcación explícita de plural en bribri implica, evidentemente, que tampoco se especifica morfológicamente el rasgo [contable] de estos referentes contables. En consecuencia, el contexto adquiere una mayor importancia en este sentido que en español, lengua en la que un referente contable carece de marcación morfosintáctica de tal propiedad únicamente cuando es determinado por un elemento anafórico o deíctico, como vimos en la sección 3 .

Ahora bien, aunque la raison d'être de los clasificadores en bribri no es la individualización del referente nominal, sí es un hecho que aparecen obligatoriamente al utilizar un numeral y que funcionan como el núcleo semántico del sintagma cuantificado, características que parecen ser los residuos de una etapa anterior de este idioma en la cual los clasificadores exhibían menor grado de gramaticalización. A modo de comparación, el español impone una restricción sintáctica muy parecida -en el ámbito verbal- que consiste en que un numeral no puede cuantificar directamente al predicado para denotar la cantidad de eventos, como en (37a), ya que este se interpretaría como parte de un sintagma nominal en función de objeto directo cuyo sustantivo viene implícito, por ejemplo manzanas o confites. Por eso, se requiere un sustantivo de por medio, típicamente veces, como en (37b). La función de este sustantivo es básicamente la misma que la de los clasificadores numerales en bribri.

(37) a. Ayer comí cuatro.

b. Ayer comí cuatro veces. 
En bribri tampoco se permite este tipo de cuantificación directa del predicado y, de hecho, en esta lengua se emplea exactamente la misma estrategia que con los sustantivos: un clasificador. En el caso de los predicados, se utiliza un clasificador numeral que no hemos mencionado anteriormente, por el hecho de que su función se encuentra fuera del ámbito nominal; este presenta la forma -kicha y podemos denominarlo VECES. Dicho sufijo se distingue, por lo tanto, de los anteriormente mencionados por siempre tener un referente verbal, no nominal. Su uso se aprecia en (38).

(38) Chakì ye' chka' tchếtökicha. ayer $1 \mathrm{SG}$ comer-PRM cuatro-CLVEC 'Ayer comí cuatro veces.'

\section{Conclusiones}

En este análisis de la contabilidad nominal en español y bribri hemos dado cuenta de las estrategias morfosintácticas que le indican al oyente si el hablante conceptualiza el referente como discreto o continuo. Este tipo de interpretación de la morfosintaxis como una proyección de la semántica constituye una aproximación nueva para una lengua de clasificadores como el bribri. Los resultados que nos brinda el punto de vista funcional tipológico se pueden resumir en la observación de que en ambos idiomas, cualquier sustantivo puede ser tanto contable como incontable, dependiendo de su entorno morfosintáctico. Por lo tanto, los sustantivos en las lenguas de clasificadores numerales parecen asemejarse, semánticamente, mucho más a sus contrapartes en otras lenguas en comparación con lo que afirman los lingüistas quienes presuponen que el valor del rasgo [contable] se encuentra especificado en las entradas léxicas de los sustantivos.

La principal diferencia en cuanto a la contabilidad nominal en las dos lenguas estudiadas es que en bribri, una mayor cantidad de sustantivos se deja sin especificar el valor del rasgo [contable], como consecuencia de las restricciones por las que se encuentra sometida la marcación de plural en esta lengua, además del hecho de que los numerales no cuantifican directamente a los nombres. En consecuencia, el contexto juega un papel más importante en el bribri que en el español a la hora de interpretar esta propiedad semántica. Sin embargo, también en español se da, aunque con menor frecuencia, que un sustantivo conceptualizado como contable se presente sin ninguna indicación gramatical de tal rasgo.

\begin{tabular}{|c|c|}
\hline- & Linde de morfema \\
\hline . & $\begin{array}{l}\text { Separa significados o elementos que en } \\
\text { conjunto traducen un solo elemento }\end{array}$ \\
\hline $1 \mathrm{SG}$ & $\begin{array}{l}\text { Sustantivo personal de primera persona } \\
\text { del singular }\end{array}$ \\
\hline $2 \mathrm{SG}$ & $\begin{array}{l}\text { Sustantivo personal de segunda persona } \\
\text { del singular }\end{array}$ \\
\hline CLALA & Clasificador ALARGADO \\
\hline CLHUM & Clasificador HUMANO \\
\hline CLPAQ & Clasificador PAQUETE \\
\hline CLPLA & Clasificador PLANO/ABSTRACTO \\
\hline CLPUÑ & Clasificador PUÑO \\
\hline CLRBA & Clasificador RACIMO DE BANANO \\
\hline CLRED & Clasificador REDONDO \\
\hline CLPES & Clasificador UNIDAD DE PESO \\
\hline CLVEC & Clasificador VECES \\
\hline ERG & Posposición que marca el ergativo \\
\hline EST & Estativo \\
\hline IMP & Imperfectivo \\
\hline PL & Plural \\
\hline PRC & Perfecto reciente \\
\hline PRM & Perfecto remoto \\
\hline SG & Singular \\
\hline
\end{tabular}




\section{Notas}

1. La gran mayoría de los hablantes del bribri habita en Costa Rica; en el Censo Nacional de Población y Vivienda 2011 de Costa Rica, alrededor de 7000 personas se declararon hablantes del bribri. Además, el Censo Nacional de Población y Vivienda 2010 de Panamá muestra que unos 1000 habitantes de ese país se consideran parte del pueblo bribri, pero el reporte no incluye el número de hablantes de la lengua.

2. Se incluye el artículo indefinido en (1c-d) justamente porque este es un marcador sintáctico de contabilidad.

3. De hecho, en algunas lenguas, los sustantivos semánticamente incontables se expresan siempre en plural (Allan 1980: 542).

4. En este artículo se hace uso de la ortografía bribri empleada por Jara y García (2013).

5. A este respecto es importante señalar que tanto Yi (2009) como Zhang (2012) presentan una visión generativista de la contabilidad como un rasgo codificado como parte de las entradas léxicas de los sustantivos.

6. En las construcciones estativas que incluyen un sustantivo incontable, se utiliza el verbo tso ${ }^{\circ} \sin$ ningún posicional.

7. Cabe agregar que también en español, los adjetivos delimitadores aparecen únicamente con sustantivos que denotan entidades individualizadas. No obstante, en esa lengua, un sintagma nominal que incluye un adjetivo prácticamente siempre presenta también algún denumerador y/o marcación morfológica de plural, por lo que el adjetivo difícilmente sería el único marcador de la contabilidad. Por tal razón, no se trató el tema en la sección 3.

\section{Bibliografía}

Allan, Keith. (1977). “Classifiers.” Language, 53 (2): 285-311.

(1980). "Nouns and countability." Language, 56 (3): 541-567.
Bach, Emmon. (1986). "The algebra of events." Linguistics \& Philosophy, 9: 5-16.

Bale, Alan y David Barner. (2009). "The interpretation of functional heads: Using comparatives to explore the mass/count distinction." Journal of Semantics, 26: 217-252.

Becerra Parra, Rodrigo. (2011). "Una propuesta funcional y tipológica de descriptores sintácticos para el mapudungun." Alpha, 32: $111-125$.

Borer, Hagit. (2005). Structuring sense. Vol. I: In name only. Oxford: Oxford University Press.

Bunt, Harry C. (1976). "The formal semantics of mass terms”. En: Karlsson (1976): 81-94.

(1985). Mass terms and model-theoretic semantics. Cambridge: Cambridge University Press.

Instituto Nacional de Estadística y Censo. (2010). XI Censo de Población y VII de Vivienda 2010. Ciudad de Panamá: Instituto Nacional de Estadística y Censo.

Instituto Nacional de Estadística y Censos. (2011). X Censo Nacional de Población y VI de Vivienda 2011. San José: Instituto Nacional de Estadística y Censos.

Chierchia, Gennaro. (1998). "Plurality of mass nouns and the notion of 'semantic parameter’.’ En: Rothstein (1998): 53-103.

Constenla Umaña, Afolfo, Feliciano Elizondo Figueroa y Francisco Pereira Mora. (1998). Curso básico de bribri. San José: Editorial de la Universidad de Costa Rica.

Corbett, Greville G. (2000). Number. Cambridge: Cambridge University Press. 
Doetjes, Jenny. (1996). "Mass and count: syntax or semantics?" Proceedings of Meaning in the HIL, HIL Occasional Papers in Linguistics, 1: 34-52.

. (1997). Quantifiers and selection. Tesis doctoral. Universidad de Leiden.

Gillon, Brendan. (1992). “Towards a common semantics for English count and mass nouns." Linguistics and Philosophy, 15 (6): 597-639.

(1999). "The lexical semantics of English count and mass nouns." En: Viegas (comp.).

Givón, Talmy. (1983a). "Topic continuity in discourse: An introduction.” En: Givón (comp.): 1-41.

Givón, Talmy (ed.) (1983b). Topic continuity in discourse. A quantitative cross language study. Ámsterdam: John Benjamins.

Givón, Talmy. (1995). Functionalism and grammar. Ámsterdam: John Benjamins.

. (2001a). Syntax. Vol. I. Ámsterdam: John Benjamins.

(2001b). Syntax. Vol. II. Ámsterdam: John Benjamins.

(2009). The genesis of syntactic complexity: Diachrony, ontogeny, neurocognition, evolution. Ámsterdam: John Benjamins.

Greenberg, Joseph H. (1963). "Some universals of grammar with particular reference to the order of meaningful elements." En: Greenberg (1963): 73-113.

Greenberg, Joseph H. (1963). Universals of human language. Londres: MIT Press.
(1974). "Numeral classifiers and substantival number: Problems in the genesis of a linguistic type." En: Proceedings of the 11th International Congress of Linguists, Boloña/Florencia, agosto/septiembre 1972: 17-37.

Grinevald, Colette. (2000). "A morphosyntactic typology of classifiers." En: Senft (2000), p. $50-92$.

Jackendoff, Jack. (1991). "Parts and boundaries." Cognition, 41: 9-41.

Jara Murillo, Carla Victoria. (1993). I ttè. Historias bribris. San José: Editorial de la Universidad de Costa Rica.

Jara Murillo, Carla Victoria y Alí García Segura. (2013). Se' ttö bribri ie. Hablemos en bribri. San José: E-Digital.

Jenner, Philip N., Laurence C. Thompson y Stanley Starosta (eds.). (1976). Austroasiatic Studies II. Oceanic Studies Special Publication No. 13. Honolulu: The University Press of Hawaii.

Jespersen, Otto. (1924). The philosophy of grammar. Londres: Allan and Unwin.

Karlsson, Fred (ed.). (1976). Papers from the 3rd Scandinavian Conference on Linguistics. Turku: Academy of Finland.

Krohn, Haakon Stensrud. (2014). “Semántica de los clasificadores numerales en el bribri de Coroma." Estudios de Lingüistica Chibcha, 33: 209-239.

Langacker, Ronald W. (1987). Foundations of cognitive grammar. Volume 1. Theoretical prerequisites. Stanford: Stanford University Press. 
(1991). Foundations of cognitive grammar. Volume 2. Descriptive application. Stanford: Stanford University Press.

Martín Arista, Javier. (1997). “La adecuación psicológica de la Gramática Funcional Tipológica." Cuadernos de Filología Inglesa, 6(2): 279-299.

Massam, Diane (ed.). (2012). A cross linguistic exploration of the count mass distinction. Oxford: Oxford University Press.

Pelletier, Francis Jeffry. (1975). "Nonsingular reference: some preliminaries". Philosophia, 5 (4): 451-465.

. (2012). "Lexical nouns are both +mass and + count, but they are neither +mass nor +count”. En: Massan, : 9-26.

Quine, William Van Orman. (1960). Word and object. Cambridge, Massachusetts: MIT Press.

Real Academia Española. (2010). Gramática de la lengua española. Manual. Madrid: Espasa.

Rothstein, Susan (ed.). (1998). Events and grammar. Dordrecht: Kluwer.
Rothstein, Susan. (2010). "Counting and the mass-count distinction." Journal of Semantics, 27 (3): 343-397.

Senft, Gunter (ed.). (2000). Systems of nominal classification. Cambridge: Cambridge University Press.

T'sou, Benjamin K. (1976). "The structure of nominal classifier systems." En: Jenner et al. (comp.): 1215-1247.

Viegas, Evelyne (ed.). (1999). Breadth and depth of semantic lexicons. Santa Cruz: Kluwer Academic.

Wierzbicka, Anna. (1988). The semantics of grammar. Ámsterdam/Filadelfia: John Benjamins Publishing Company.

Yi, Byeong-Uk. (2009). "Chinese classifiers and count nouns." Journal of Cognitive Science, 10 (2): 209-226.

Zhang, Niina Ning. (2012). Numeral Classifier Structures. Manuscrito no publicado, Universidad Nacional Chung Cheng, Minxiong, Taiwán. Disponible en: ling. auf.net/lingbuzz/001197/v3.pdf

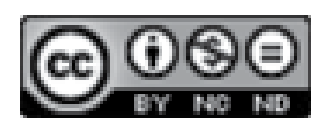

Este obra está bajo una licencia de Creative Commons

Reconocimiento-NoComercial-SinObraDerivada 4.0 Internacional. 